\title{
A novel method to accurately locate the reconstructed auricle
}

\author{
Pengfei Sun, Changchen Wang, Xin Huang, Bo Pan^ \\ Department of Auricular Reconstruction, Plastic Surgery Hospital, Chinese Academy of Medical Sciences and Peking Union Medical College, \\ Beijing, China \\ Contributions: (I) Conception and design: P Sun, B Pan; (II) Administrative support: P Sun, B Pan; (III) Provision of study materials or patients: P \\ Sun, C Wang, X Huang; (IV) Collection and assembly of data: P Sun, C Wang; (V) Data analysis and interpretation: P Sun, C Wang, X Huang; (VI) \\ Manuscript writing: All authors; (VII) Final approval of manuscript: All authors. \\ Correspondence to: Bo Pan, MD. Department of Auricular Reconstruction, Plastic Surgery Hospital, Chinese Academy of Medical Sciences and Peking \\ Union Medical College, No. 33 Badachu Road, Shijingshan District, Beijing 100144, China. Email: zbzbzhc@163.com.
}

\begin{abstract}
Background: Congenital microtia is a common congenital disease in children, the cause of which is still unclear. At present, the main treatment for congenital microtia is ear reconstruction. Accurately locating of the reconstructed ear on the affected side before ear reconstruction surgery is difficult, while it is the key of successful operation. Our ear reconstruction team has developed a novel method to accurately locate the reconstructed auricle. This novel method has achieved good results in clinical practice.
\end{abstract}

Methods: Thirty patients with unilateral ear reconstruction, who underwent auricle reconstruction using our invented auricle reconstruction positioning method in the Plastic Surgery Hospital of Chinese Academy of Medical Sciences from January 2020 to July 2021, were enrolled in this study.

Results: Through Wilcoxon signed rank test, we found that there was no statistical difference between the mean distance from the highest point of the patient's normal ear to the central axis of the nose and that from the highest point of the reconstructed ear to the central axis of the nose $(\mathrm{P}>0.05)$. Meanwhile, there was no statistical difference between the mean distance from the lowest point of the patient's normal ear to the central axis of the nose and that from the lowest point of the reconstructed ear to the central axis of the nose $(\mathrm{P}>0.05)$. The satisfaction rate of patients and their families to the location of the reconstructed auricle was $100 \%$.

Conclusions: The novel method of locating the reconstructed auricle employs simple materials. The implementation process is easy, and the effect is significant. To a certain extent, it solves the difficulty of locating the reconstructed auricle in ear reconstruction operation. Although this method can only be applied to patients with unilateral microtia, we recommend it for locating the reconstructed auricle by every plastic surgeon.

Keywords: Reconstructed auricle; ear reconstruction; auricle location; congenital microtia

Submitted Sep 16, 2021. Accepted for publication Jan 28, 2022.

doi: $10.21037 /$ tp-21-453

View this article at: https://dx.doi.org/10.21037/tp-21-453

\section{Introduction}

Congenital microtia is a common congenital disease in children, the cause of which is still unclear (1). The clinical symptoms of congenital microtia are mainly auricle dysplasia, which is usually accompanied by external auditory canal atresia, middle ear and maxillofacial malformation (2). At present, the main treatment for congenital microtia is ear reconstruction (3). In China, auricle reconstruction with autogenous rib cartilage scaffold is the mainstream method for the treatment of congenital microtia (4). A symmetrically sized ear is one of the criteria for successful ear reconstruction (5). Therefore, accurately locating the reconstructed ear on the affected side before ear reconstruction surgery is the key of successful operation.

^ ORCID: 0000-0001-8900-8479. 


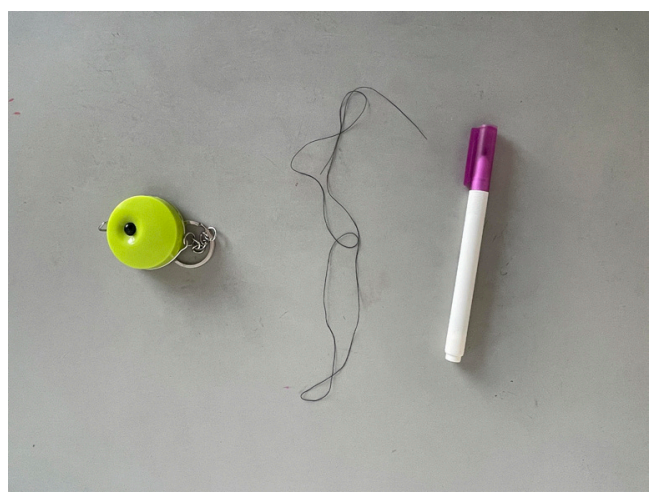

Figure 1 The materials used in the novel method.

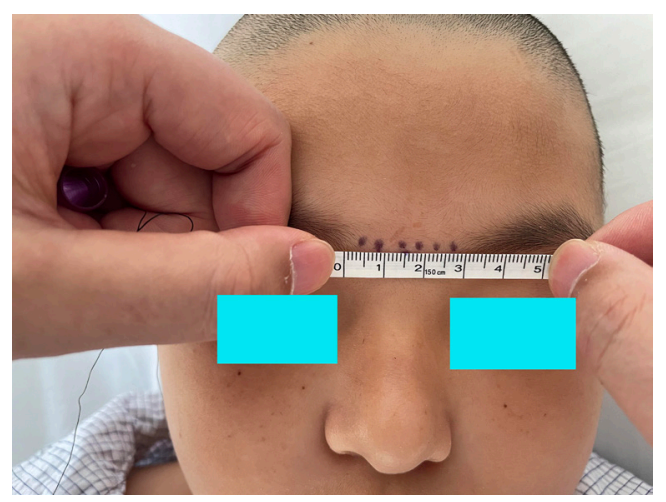

Figure 2 Locating the midpoint of the line between the two brows. This image is published with consent from the patient's legal guardian.

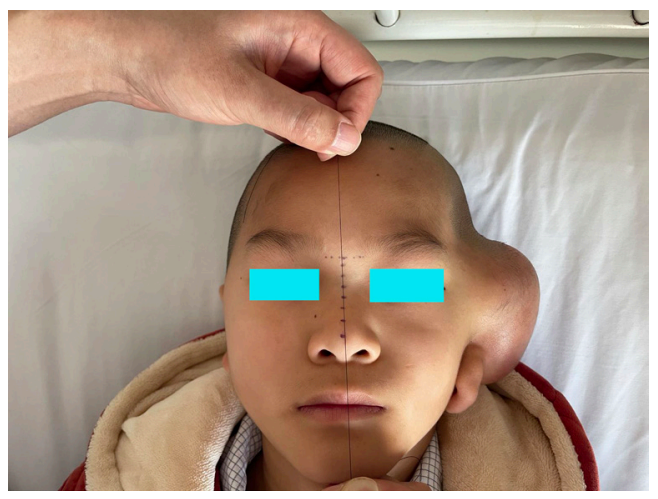

Figure 3 Drawing the central axis of the patient's nose. This image is published with consent from the patient's legal guardian.
However, there is still no unified standard for locating reconstructed auricle in clinical practice (6). In addition, the accurate location of the reconstructed auricle is also one of the difficulties for ear reconstruction (7). At present, the location of reconstructed auricle in clinical practice is mostly determined by the experience of plastic surgeons. Moreover, there is a lack of clinical studies on the location of reconstructed auricle. Based on the experience in auricle reconstruction, our ear reconstruction team has developed a novel method of locating the reconstructed auricle, which has achieved good results in clinical practice. We present the following article in accordance with the STROBE reporting checklist (available at https://tp.amegroups.com/article/ view/10.21037/tp-21-453/rc) (8).

\section{Methods}

\section{Clinical data}

Thirty patients with unilateral ear reconstruction, who underwent auricle reconstruction using our invented auricle reconstruction positioning method in the Plastic Surgery Hospital of Chinese Academy of Medical Sciences from January 2020 to July 2021, were enrolled in this study. Among them, 18 were males and 12 were females, with ages of 6-16 years old. Right ear reconstruction was performed in 13 cases and left ear reconstruction was performed in 17 cases. The study was conducted in accordance with the Declaration of Helsinki (as revised in 2013). This study was approved by the Medical Ethics Committee of Plastic Surgery Hospital of the Chinese Academy of Medical Sciences (No. 2021184). Patients' parents or legal guardians signed informed consent.

\section{Locating reconstructed ear}

(I) The materials used in this method included a ruler, a cotton thread and a drawing pen (Figure 1);

(II) The doctor connected patient's two brows to locate the midpoint of the line between the two brows (Figure 2). The doctor used a straightened cotton thread to draw the central axis of the patient's nose by crossing the midpoint perpendicularly with the midpoint of the line between the two brows (Figure 3);

(III) The doctor straightened a cotton thread to vertically intersect with the central axis of the patient's nose, and determined the position of the reconstructed ear upper edge according to the upper edge of the 


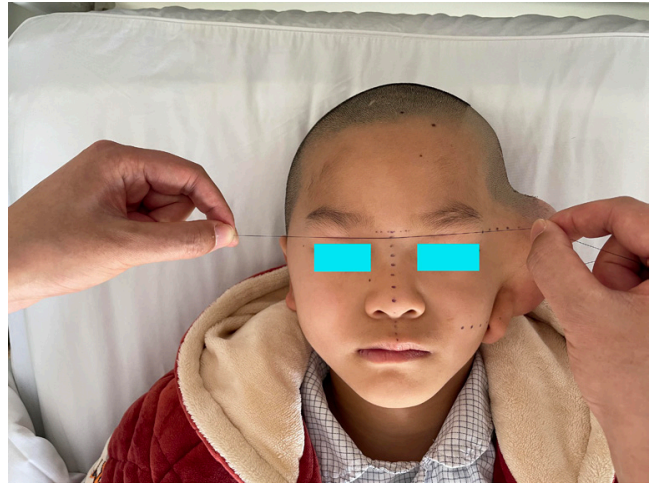

Figure 4 Determining the position of the reconstructed ear upper edge. This image is published with consent from the patient's legal guardian.

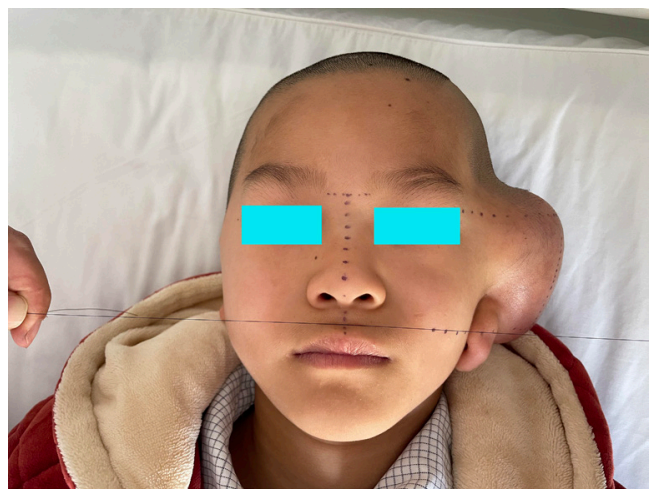

Figure 5 Determining the position of the reconstructed ear lower edge. This image is published with consent from the patient's legal guardian.

patient's healthy lateral ear (Figure 4);

The doctor used a straightened cotton thread to vertically intersect with the central axis of the patient's nose, and determined the position of the lower edge of the reconstructed earlobe according to the horizontal line of the lower edge of the patient's healthy earlobe (Figure 5);

(V) The doctor used a cotton thread to determine the position of the midpoint of the tragus of the reconstructed ear based on the vertical distance from the midpoint of the tragus on the healthy side to the central axis of the nose (Figures 6,7);

(VI) The doctor used a straightened cotton thread to determine the lowest point of the reconstructed earlobe according to the vertical distance from the lowest point of the patient's healthy earlobe to the

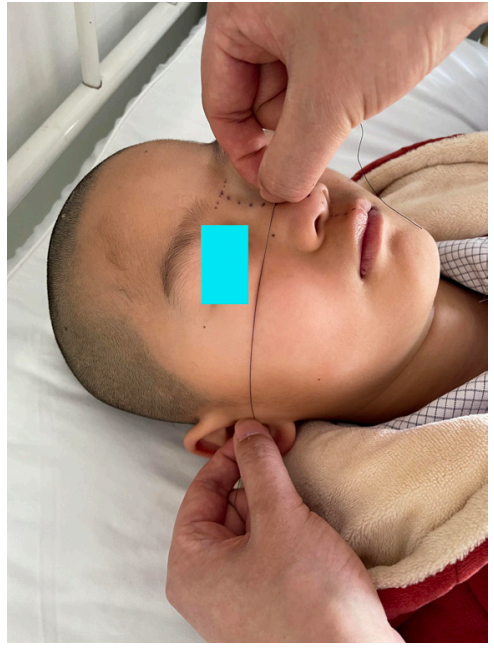

Figure 6 Measuring the vertical distance from the midpoint of the tragus on the healthy side to the central axis of the nose. This image is published with consent from the patient's legal guardian.

central axis of the patient's nose (Figures 8,9);

The doctor connected the lowest point of the reconstructed ear lobe and the midpoint of the reconstructed tragus by a straight line. This straight line and the straight lines of the upper and lower edges of the reconstructed ear determined the scope of the reconstructed ear (Figure 10);

(VIII) The tilt angle of the reconstructed ear was determined according to the principle that the long axis of the reconstructed ear was parallel to the central axis of the patient's nose.

This novel method of locating the reconstructed auricle was performed by two surgeons, for which one surgeon took measurements, and the other one drew lines by using a marker. The location of the reconstructed auricle was performed before surgery. The surgeon performed auricle reconstruction according to the preoperative marking lines.

\section{Effect evaluation}

We compared the mean distance from the highest point of the patient's normal ear to the central axis of the nose with that from the highest point of the reconstructed ear to the central axis of the nose. Meanwhile, the mean distance from the lowest point of the patient's normal ear earlobe to the central axis of the nose was compared with that from the lowest point of the reconstructed ear earlobe to the central axis of the nose. The symmetry between the reconstructed 


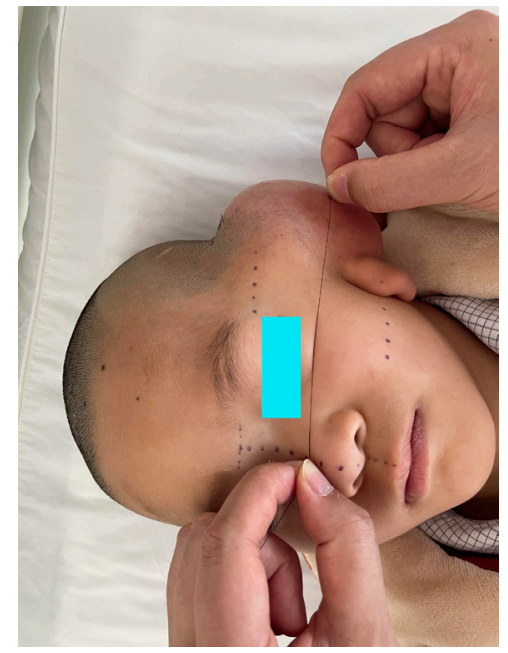

Figure 7 Determining the position of the midpoint of the tragus of the reconstructed ear. This image is published with consent from the patient's legal guardian.

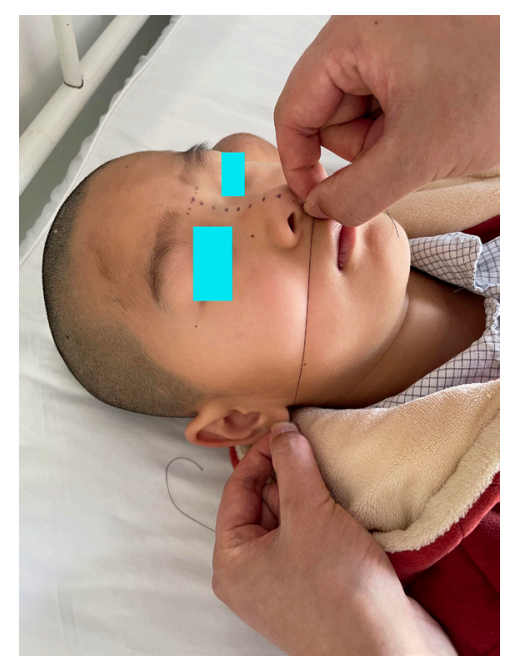

Figure 8 Measure the vertical distance from the lowest point of the patient's healthy earlobe to the central axis of the patient's nose. This image is published with consent from the patient's legal guardian.

ear and the normal ear was evaluated by comparing the two sets of data. Then, the effectiveness of the novel auricle positioning method developed by our ear reconstruction team was evaluated according to the symmetry of the patient's two ears. Questionnaire survey was used to obtain the satisfaction rate of patients and their families on the location of the reconstructed ear.

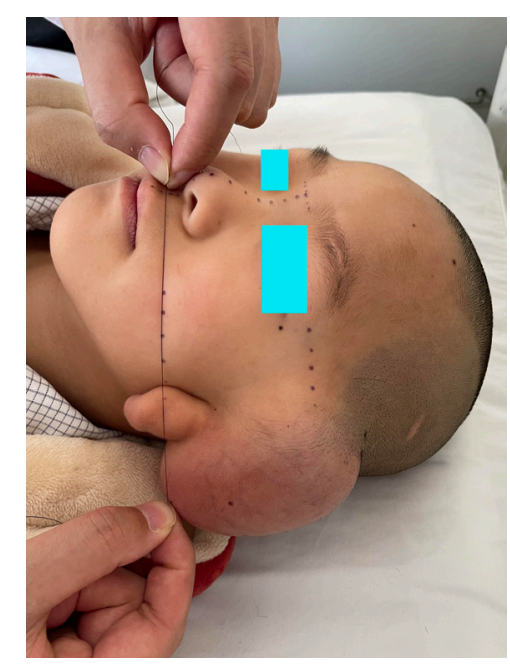

Figure 9 Determining the lowest point of the reconstructed earlobe. This image is published with consent from the patient's legal guardian.

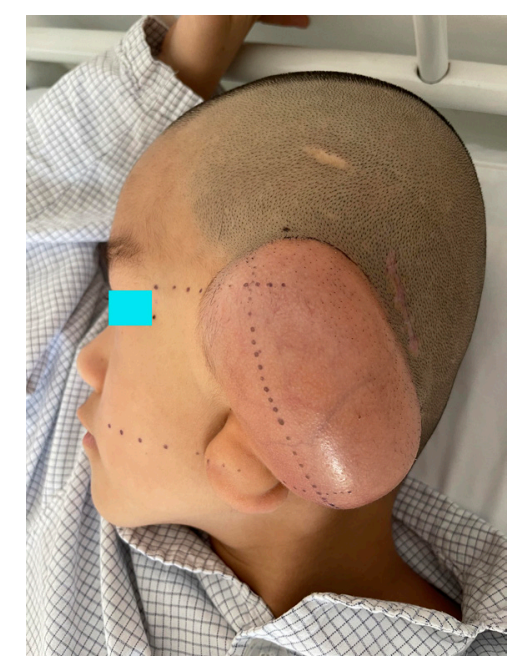

Figure 10 Determining the scope of the reconstructed ear. This image is published with consent from the patient's legal guardian.

\section{Statistical analysis}

Continuous variables were represented by mean \pm standard deviation (SD), and classified variables were represented by number of cases and percentage. We used the SPSSAU data scientific analysis platform (https://spssau.com) for data analysis. Wilcoxon signed rank test was used for statistical analysis of the main evaluation criteria, and the 
Table 1 Data summary

\begin{tabular}{|c|c|c|}
\hline Variables & Specific values & Wilcoxon signed rank test, $\mathrm{P}$ value \\
\hline \multicolumn{3}{|l|}{ Gender } \\
\hline Male & $18(60 \%)$ & - \\
\hline Female & $12(40 \%)$ & - \\
\hline $\begin{array}{l}\text { The mean distance between the highest point of the helix of the normal ear and } \\
\text { the central axis of the nose, } \mathrm{cm}\end{array}$ & $14.23 \pm 0.64$ & $0.31(P>0.05)$ \\
\hline $\begin{array}{l}\text { The mean distance between the highest point of the helix of the reconstructed } \\
\text { ear and the central axis of the nose, } \mathrm{cm}\end{array}$ & $14.2 \pm 0.56$ & \\
\hline \multicolumn{3}{|l|}{ Satisfaction rate of patients and their families } \\
\hline Great satisfaction & $24(80 \%)$ & - \\
\hline Satisfaction & $6(20 \%)$ & - \\
\hline Unsatisfaction & 0 & - \\
\hline
\end{tabular}

value of $\mathrm{P}<0.05$ was set as statistical significance for data comparison.

\section{Results}

In our study, the mean age of the patients was $10.5 \pm 3.13$ years old. The mean follow-up time was $7.5 \pm 2.15$ months. The mean distance between the highest point of the helix of the normal ear and the central axis of the nose was $14.23 \pm 0.64 \mathrm{~cm}$. The mean distance between the highest point of the helix of the reconstructed ear and the central axis of the nose was $14.2 \pm 0.56 \mathrm{~cm}$. The mean distance between the lowest point of the earlobe of the normal ear and the central axis of the nose was $12.84 \pm 0.59 \mathrm{~cm}$. The mean distance between the lowest point of the earlobe of the reconstructed ear and the central axis of the nose was $12.83 \pm 0.5 \mathrm{~cm}$. Through Wilcoxon signed rank test, we found that no statistical difference between the mean distance from the highest point of the patient's normal ear to the central axis of the nose and that from the highest point of the reconstructed ear to the central axis of the nose $(\mathrm{P}>0.05)$. Meanwhile, there was no statistical difference between the mean distance from the lowest point of the patient's normal ear to the central axis of the nose and that from the lowest point of the reconstructed ear to the central axis of the nose $(\mathrm{P}>0.05)$. This result indicated that the patients' normal ears and the reconstructed ears were symmetrical. Through the questionnaire survey, we found that the satisfaction rate of patients and their families to the location of the reconstructed auricle was 100\% [Table 1, the data is available in the supplementary file (available at https:// cdn.amegroups.cn/static/public/tp-21-453-01.xls)].

The typical cases were shown in Figures 11-16.

\section{Discussion}

At present, most of the studies on auricle reconstruction focused on the surgical methods of auricle reconstruction and auricle scaffold materials used in auricle reconstruction (9-11). The researchers have ignored the importance of accurate positioning of the reconstructed auricle. Auricle reconstruction can achieve high effectiveness only when the reconstructed auricle and the healthy auricle were symmetrical (12). The position of the auricle on the healthy side and the auricle on the affected side in patients with microtia was often inconsistent, and some patients were accompanied by maxillofacial deformity (13). Meanwhile, in clinical practice, plastic surgeons cannot find an effective way to accurately locate the reconstructed auricle (14). 


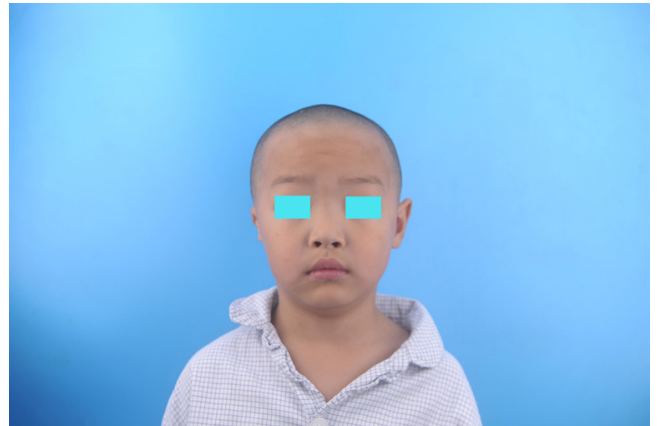

Figure 11 Preoperative frontal view of patient 1 . This image is published with consent from the patient's legal guardian.

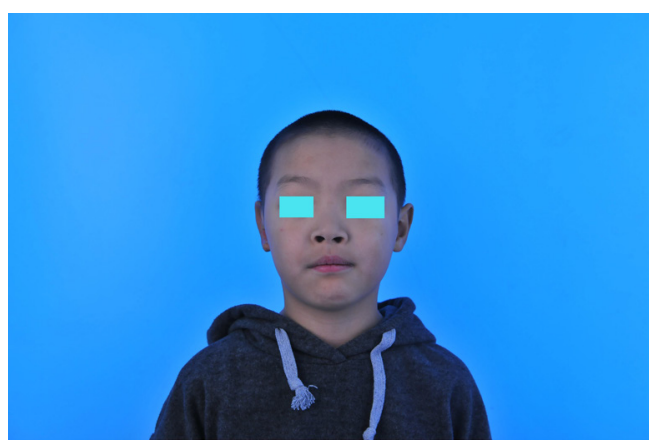

Figure 12 Postoperative frontal view of patient 1 . This image is published with consent from the patient's legal guardian.

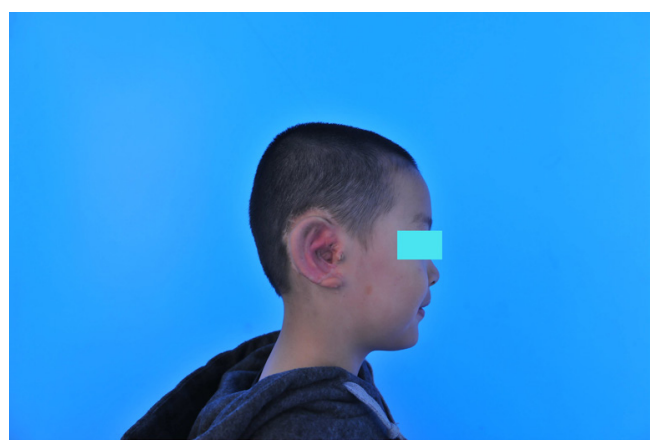

Figure 13 Postoperative lateral view of patient 1. This image is published with consent from the patient's legal guardian.

Therefore, accurately locating the position of reconstructed auricle has always been a difficult problem for plastic surgeons $(15,16)$.

The method of locating the reconstructed auricle used in this study was invented by our ear reconstruction team. The materials used in this method included a ruler, a

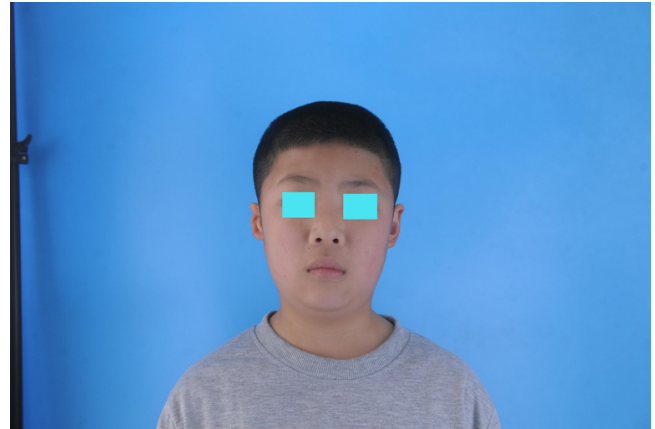

Figure 14 Preoperative frontal view of patient 2. This image is published with consent from the patient's legal guardian.

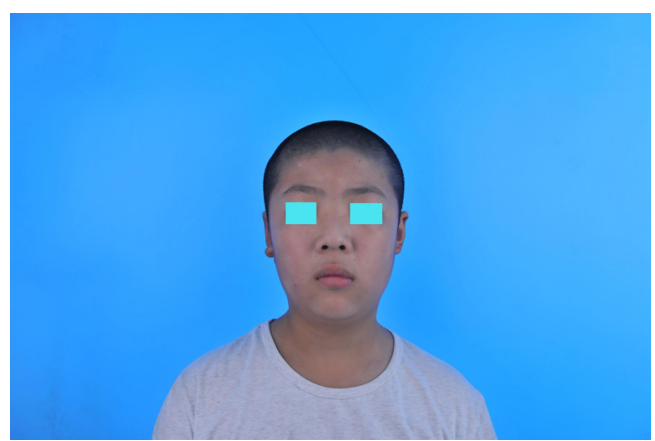

Figure 15 Postoperative frontal view of patient 2. This image is published with consent from the patient's legal guardian.

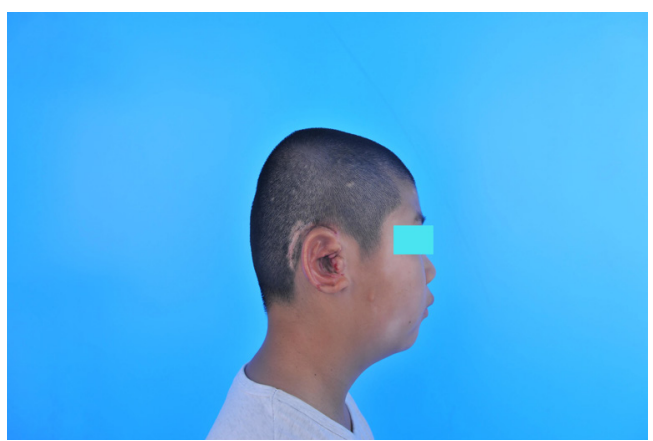

Figure 16 Postoperative lateral view of patient 2. This image is published with consent from the patient's legal guardian.

cotton thread and a drawing pen. This method mainly took the central axis of the patient's nose as a reference, and determined the upper edge of the reconstructed auricle, the lower edge of the reconstructed auricle, the midpoint of the tragus of the reconstructed auricle and the lowest point of the earlobe of the reconstructed auricle through the vertical 
intersection of the straightened cotton thread and the central axis of the patient's nose. The tilt angle of the reconstructed ear was determined by the long axis of the reconstructed ear parallel to the central axis of the nose. Postoperative photographs of the 30 patients showed that this novel method of locating the reconstructed auricle was very effective.

When applying this newly invented the reconstructed auricle positioning method invented by us, the following points need to be paid attention: (I) the cotton thread must be straightened. Otherwise, the accurate position of the reconstructed auricle will be affected; (II) it is important to determine the exact position of the central axis of the nose, because the central axis of the nose is the important reference for determining the upper edge of the reconstructed auricle, the lower edge of the reconstructed auricle, the tragus midpoint of the reconstructed auricle and the lowest point of the earlobe of the reconstructed auricle; (III) this novel method is only applicable to patients undergoing unilateral ear reconstruction, because we determined the location of the reconstructed auricle according to the location of the patient's healthy ear; (IV) this novel method requires the cooperation of two doctors: one is responsible for measuring using a straightened cotton thread, and the other is responsible for drawing lines to determine the exact location of the reconstructed auricle; (V) this novel method of locating the reconstructed auricle can only be completed with the cooperation of the patient; or the positioning of auricle reconstruction can be carried out after the patient is asleep or anesthetized on the operating table; (VI) in clinical practice, for patients with microtia that are accompanied by unilateral facial microsomia, the corresponding adjustment when using cotton thread to locate the reconstructed auricle should be performed according to the condition of the patient's face, so that the reconstructed ear is in harmony with the facial morphology.

In terms of evaluating the effectiveness of this novel method, we compared the mean distance from the highest point of the patient's normal ear to the central axis of the nose with that from the highest point of the reconstructed ear to the central axis of the nose. The mean distance from the lowest point of the patient's normal ear earlobe to the central axis of the nose was also compared with that from the lowest point of the reconstructed ear earlobe to the central axis of the nose. These two indicators were used to assess the symmetry between the reconstructed ear and the normal ear, which is simple but not precise enough. In 2021, Hallac et al. $(17,18)$ invented the method of using the Convolutional Neural Network to evaluate auricle morphology, and in 2020, Mussi et al. (19) invented the computer-aided strategy for preoperative simulation of autologous ear reconstruction procedure. We can apply these two methods in future studies to evaluate the morphology of the reconstructed auricle and the symmetry between the reconstructed auricle and the normal ear, so as to evaluate the positioning effect of the novel method of positioning the reconstructed auricle more objectively.

\section{Conclusions}

The novel method of locating the reconstructed auricle uses simple materials, the implementation process is easy, and the effect is significant. To a certain extent, it solves the difficulty of locating the reconstructed auricle in ear reconstruction operation. Although this method is only suitable for patients with unilateral microtia, we recommend it for locating the reconstructed auricle by every plastic surgeon.

\section{Acknowledgments}

The authors thank all the patients for their participation in this study. We would also like to thank TopEdit (www. topeditsci.com) for its linguistic assistance during the preparation of this manuscript.

Funding: This work was supported by the National Natural Science Foundation of China (NSFC) (grant No. 81571863), the Institute Foundation of Plastic Surgery Hospital, Chinese Academy of Medical Sciences (No. 3060120031), and the CAMS Innovation Fund for Medical Sciences (CIFMS) (No. 2021-12M-1-052).

\section{Footnote}

Reporting Checklist: The authors have completed the STROBE reporting checklist. Available at https:// tp.amegroups.com/article/view/10.21037/tp-21-453/rc

Data Sharing Statement: Available at https://tp.amegroups. com/article/view/10.21037/tp-21-453/dss

Peer Review File: Available at https://tp.amegroups.com/ article/view/10.21037/tp-21-453/prf

Conflicts of Interest: All authors have completed the ICMJE uniform disclosure form (available at https://tp.amegroups. com/article/view/10.21037/tp-21-453/coif). The authors 
have no conflicts of interest to declare.

Ethical Statement: The authors are accountable for all aspects of the work in ensuring that questions related to the accuracy or integrity of any part of the work are appropriately investigated and resolved. The study was conducted in accordance with the Declaration of Helsinki (as revised in 2013). This study was approved by the Medical Ethics Committee of Plastic Surgery Hospital of the Chinese Academy of Medical Sciences (No. 2021184). Patients' parents or legal guardians signed informed consent.

Open Access Statement: This is an Open Access article distributed in accordance with the Creative Commons Attribution-NonCommercial-NoDerivs 4.0 International License (CC BY-NC-ND 4.0), which permits the noncommercial replication and distribution of the article with the strict proviso that no changes or edits are made and the original work is properly cited (including links to both the formal publication through the relevant DOI and the license). See: https://creativecommons.org/licenses/by-nc-nd/4.0/.

\section{References}

1. Torres I, Martinez JDC, Sanabria R, et al. Postoperative Safety and Satisfaction in Patients With Microtia. J Oral Maxillofac Surg 2021;79:472.e1-9.

2. Zou K, Fan Y, Jiang L, et al. Ear mold for congenital ear malformation: A randomized controlled trial. Medicine (Baltimore) 2020;99:e21313.

3. Joukhadar N, McKee D, Caouette-Laberge L, et al. Management of Congenital Auricular Anomalies. Plast Reconstr Surg 2020;146:205e-16e.

4. Zhang Y, Liu J. Improve the appearance of auriculocephalic angle in reconstructed auricular with skin flap of residual ear in patients with microtia of concha cavity. Zhongguo Xiu Fu Chong Jian Wai Ke Za Zhi 2020;34:915-8.

5. Otto IA, Simone A, Malda J, et al. Engaging stakeholders in bioprinting research: Views and concerns of microtia patients' parents on bioprinted auricular cartilage. J Plast Reconstr Aesthet Surg 2020;73:2239-60.

6. Guo F, Lin L, Jiang H. Research progress of clinical therapy for concha-type microtia. Zhongguo Xiu Fu Chong Jian Wai Ke Za Zhi 2020;34:656-9.

7. Lang Z, Xiong T, Wang M, et al. A Simple and Accurate Method for the Production of a Personalized Twodimensional Auricular Template. Aesthetic Plast Surg
2020;44:1106-7.

8. von Elm E, Altman DG, Egger M, et al. The Strengthening the Reporting of Observational Studies in Epidemiology (STROBE) Statement: guidelines for reporting observational studies. Int J Surg 2014;12:1495-9.

9. Grundmann T. Surgical management of high-grade ear malformations in childhood. HNO 2020;68:401-6.

10. Chang S, Zhang Q. International Consensus Recommendations on Microtia, Aural Atresia and Functional Ear Reconstruction. J Int Adv Otol 2019;15:472-3.

11. Guo F, Lin L, Yu X, et al. Classification of the conchatype microtia and their new suitable treatment strategies without autogenous costal cartilage grafting. Int J Pediatr Otorhinolaryngol 2020;130:109801.

12. Chen K, Bradley JP. Autologous Ear Reconstruction: Challenging But Vital to Plastic Surgery. Plast Reconstr Surg 2019;144:1121e.

13. Cuccolo NG, Zwierstra MJ, Ibrahim AMS, et al. Reconstruction of Congenital Microtia and Anotia: Analysis of Practitioner Epidemiology and Postoperative Outcomes. Plast Reconstr Surg Glob Open 2019;7:e2318.

14. Nuri T, Mitsuno D, Otsuki Y, et al. Augmented Reality Technology for the Positioning of the Auricle in the Treatment of Microtia. Plast Reconstr Surg Glob Open 2020;8:e2626.

15. Zhang Z, Liu R, Wang R, et al. Application of threedimensional mechanical equilibrium concept in cartilage scaffold construction for total auricular reconstruction. Zhongguo Xiu Fu Chong Jian Wai Ke Za Zhi 2019;33:332-6.

16. Xu Z, Zhang R, Zhang Q, et al. New Strategies for Remnant Ear Treatment in Lobule-Type Microtia Reconstruction. Plast Reconstr Surg 2018;142:471-9.

17. Hallac RR, Lee J, Pressler M, et al. Identifying Ear Abnormality from 2D Photographs Using Convolutional Neural Networks. Sci Rep 2019;9:18198.

18. Hallac RR, Jackson SA, Grant J, et al. Assessing outcomes of ear molding therapy by health care providers and convolutional neural network. Sci Rep 2021;11:17875.

19. Mussi E, Servi M, Facchini F, et al. A computer-aided strategy for preoperative simulation of autologous ear reconstruction procedure. International Journal on Interactive Design and Manufacturing (IJIDeM) 2021;15:77-80.

Cite this article as: Sun P, Wang C, Huang X, Pan B. A novel method to accurately locate the reconstructed auricle. Transl Pediatr 2022;11(4):487-494. doi: 10.21037/tp-21-453 